\title{
Effect of boron and nitrogen additives on structure and transport properties of arc-produced carbon
}

\author{
O.V. Sedelnikova a, b, *, Yu.V. Fedoseeva ${ }^{\text {a }}$, A.I. Romanenko ${ }^{a}$, A.V. Gusel'nikov ${ }^{\text {a }}$,

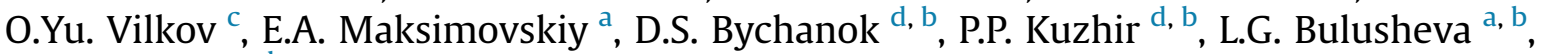 \\ A.V. Okotrub a, b \\ a Nikolaev Institute of Inorganic Chemistry, SB RAS, 3 Acad. Lavrentiev Ave., 630090, Novosibirsk, Russia \\ b Tomsk State University, 36 Lenin Ave., Tomsk, 634050, Russia \\ c St. Petersburg State University, 7/9 Universitetskaya nab., 199034, Petersburg, Russia \\ ${ }^{\mathrm{d}}$ Institute for Nuclear Problems of Belarusian State University, 11 Bobruiskaya Str., 220030, Minsk, Belarus
}

\section{A R T I C L E I N F O}

\section{Article history:}

Received 3 October 2018

Received in revised form

2 November 2018

Accepted 24 November 2018

Available online 24 November 2018

\begin{abstract}
A B S T R A C T
We have studied the effect of introduction of boron, nitrogen or both elements into an electric arc on the morphology and the conductivity of the resultant carbon products. Scanning and transmission electron microscopies showed that the use of a boron-filled graphite electrode and a nitrogen gas during the arc discharge synthesis strongly affects the growth kinetics of carbon nanoparticles. The addition of boron promotes the formation of short, defective carbon nanotubes. In contrast, involvement of nitrogen in the synthesis process produces more perfect carbon nanostructures, including graphitic plates. Evaporation of a boron-filled electrode in a nitrogen atmosphere leads to BN co-doping of the carbon product. The concentration of each dopant is ca. 1 at.\% and this value is twice greater than that for the cases of individual dopants. Among the studied materials, the BN-doped one possessed the highest conductivity, and this was attributed to the synergetic effect of co-doping. A substitution of carbon atoms by boron or nitrogen resulted in the $p$ - or $n$-type doping of the samples, respectively. The evolution of conductivity with temperature and magnetic field showed that transport properties of the arc discharge synthesis products are strongly dependent on the charge carrier concentration, morphology and crystallinity of carbon nanoparticles.
\end{abstract}

() 2018 Elsevier Ltd. All rights reserved.

\section{Introduction}

Arc-discharge is a capable process for mass production of metalfree carbon nanotubes (CNTs) [1,2]. The extremely high temperature generated in the electric arc leads to atomization of the graphitic electrode. Subsequent cooling of the gas jet gives carbon nanostructures with very little disorders that is a great advantage of the arc-discharge synthesis. However, due to the complexity of process, CNTs are formed simultaneously with other forms of carbon, such as fullerenes [3], polyhedral particles [4], graphene and nano-graphites [5]. The content of co-products depends on the synthesis parameters, such as gas type and pressure, current density, discharge power, etc. [6-8].

\footnotetext{
* Corresponding author. Nikolaev Institute of Inorganic Chemistry, SB RAS, 3 Acad. Lavrentiev Ave., 630090, Novosibirsk, Russia.
}

E-mail address: o.sedelnikova@gmail.com (O.V. Sedelnikova).
The doping of carbon nanostructures with heteroatoms, such as boron or nitrogen, is one of the effective ways to tune their properties in accordance with the particular application's requirements. Heteroatom doping can be performed either during the synthesis or by post-synthesis treatments, including ion implantation [9], plasma treatment [10], and annealing in nitrogen-containing atmosphere [11,12]. Several studies have been devoted to the synthesis of the $\mathrm{B}$ - and $\mathrm{N}$-doped carbon nanostructures using arc discharge [6,8,13-25]. These nanostructures are formed in a nitrogen atmosphere [18,19] and using boron-filled graphite electrodes [17,21,24,25]. In the co-doped deposit, boron and nitrogen are found as individual defects $[6,13,20,22]$ or $B N$ domains $[13,14,16,21]$. Some reports showed that the introduction of dopants in an electric arc results in enhancement of the crystallinity of carbon nanomaterials $[13,15,17]$, while others reported formation of more defective products [6,17,18,23]. Electronic, optical and conducting properties of the B- and N-doped multi-walled CNTs 
(MWCNTs) have been reported in Refs. [24-30]. Particularly, it was shown that even a small amount of dopant ( $<1$ at.\%) can significantly enhance the conductivity of nanotubes [24,25,28,29]. On the other hand, dopants could act as additional defects, deteriorating the conductivity [30].

The present communication aimed to explore the modification of morphology and charge transport properties of carbon materials arising from addition of nitrogen and/or boron species in the electric arc. The morphology of nanoparticles was examined by scanning electron microscopy (SEM) and transmission electron microscopy (TEM), the defect density and chemical composition were investigated by Raman spectroscopy, X-ray photoelectron spectroscopy (XPS) and near-edge X-ray absorption fine structure spectroscopy (NEXAFS). The transport properties were studied by measuring the temperature dependence of conductivity and magnetoconductivity (MC).

\section{Experimental part}

\subsection{Synthesis}

The setup used for the syntheses is described in details elsewhere [31]. Briefly, an upper movable cathode was made from a graphite rod of $60-\mathrm{mm}$ diameter. A graphite anode had a crosssection of $14 \times 14 \mathrm{~mm}$ and a length of $200 \mathrm{~mm}$. The anode was placed on a water-cooled holder in bottom of the chamber opposite the cathode. All syntheses were carried out at arc current of $500 \mathrm{~A}$, arc voltage of $50 \mathrm{~V}$ and gas pressure of $10^{5} \mathrm{~Pa}$, which have been proved to be the optimal parameters for growth of MWCNTs [7,25]. The synthesis duration was $25 \mathrm{~min}$.

Undoped and $\mathrm{N}$-doped carbon samples were produced by evaporation of solid graphite rod in helium and nitrogen atmosphere, respectively. To produce B-doped and BN co-doped samples, a cylindrical cavity in central part of the rod was filled by powder of amorphous boron mixed with graphite powder in a ratio of $1: 1$. The concentration of boron in the rod was $c a .5 \mathrm{wt} . \%$. The electrode was evaporated in helium or in nitrogen atmosphere to produce either B-doped or BN-doped samples.

The arc discharge evaporation of anodes produces dense deposits on the cathode surface and soot on the water-cooled walls of the chamber. The initial weight of the anodes was $c a .70 \mathrm{~g}$, the mass of the deposits was $\mathrm{ca} .10 \mathrm{~g}$. The samples for the investigations were taken from central part of the deposits. Although the storage of a carbon sample in laboratory conditions usually results in $p$-type doping by atmospheric oxygen [32], further, we will refer the pure carbon material as "undoped" sample meaning that it does not contain nitrogen or boron impurities.

\subsection{Methods}

The morphology of samples was examined by SEM on a JEOL JSM 6700F microscope and TEM on a JEOL 2010 microscope. Raman spectra were collected with a Spex 1877 triple spectrometer using the 488-nm line from an argon laser. XPS and NEXAFS spectra were recorded at the Berliner Elektronenspeicher ring für Synchrotronstrahlung (BESSY II) using radiation from the Russian-German beamline. A powder sample was attached to a scratched copper substrate and placed into a chamber maintained at a pressure of $10^{-7} \mathrm{~Pa}$. The XPS spectra were measured at a monochromatic radiation of $800 \mathrm{eV}$. After subtraction of a Shirley background, the C, $\mathrm{N}$, and B 1s XPS spectra were fitted using Gaussian/Lorentzian functions within Casa XPS 2.3.15 software. The NEXAFS spectra near $\mathrm{C}-, \mathrm{N}-$, and B K-edge were recorded in the total-electron yield mode with an experimental resolution better than $0.1 \mathrm{eV}$.

The direct current (DC) conductivity $\sigma$ measurements of parallelogram-shaped samples $(1 \times 1 \times 5 \mathrm{~mm})$ were carried out using afour-probe technique in a temperature range of $4.2-292 \mathrm{~K}$. The Hall effect and MC were measured by an eight-contact method at the temperature of liquid helium $(4.2 \mathrm{~K})$. The $\mathrm{MC}$ was defined as $\frac{\sigma(H)}{\sigma(0)}-1$, where $\sigma(0)$ and $\sigma(H)$ are the DC conductivity without and with the magnetic field $H$ applied, respectively.

\section{Results and discussion}

\subsection{Characterization}

Typical SEM and TEM images of the samples are presented in Figs. 1 and 2, respectively. All samples are highly nonhomogeneous. They consist of straight MWCNTs, polyhedral nanoparticles and graphitic plates of different shapes and sizes. The simultaneous growth of nanotubes together with other co-products is typical for the arc discharge process [1,7]. Involvement of nitrogen or boron in the synthesis influences the characteristics of arc-discharge, which might differ from the optimal conditions for the nanotubes growth. The evaporation of the solid graphite rode in a nitrogen atmosphere promotes the formation of graphitic plates and strongly limits the growth of MWCNTs, whereas the B-doped and BN-doped samples are enriched with short MWCNTs and polyhedral nanoparticles. This fact suggests that the addition of amorphous boron into graphite electrode results in disordering of graphitic structures that terminates the growth of long nanotubes.

Raman spectra of the samples exhibited two peaks in the region between 1000 and $2000 \mathrm{~cm}^{-1}$ (Fig. 3), which are typical for graphite [33]. The $\mathrm{G}$ band at $1577 \mathrm{~cm}^{-1}$ is assigned to the in-plane vibrations of $\mathrm{C}-\mathrm{C}$ bonds and the $\mathrm{D}$ band at $1358 \mathrm{~cm}^{-1}$ is activated in the presence of disorder in graphite lattice. Since the intensity of D band increases with the number of defects and functionalities, the intensity ratio of $\mathrm{D}$ and $\mathrm{G}$ bands $\left(I_{\mathrm{D}} / I_{\mathrm{G}}\right)$ is commonly used to estimate structural disorder in graphitic materials. The $I_{\mathrm{D}} / I_{\mathrm{G}}$ ratio is equal to 0.2 for the undoped and $\mathrm{N}$-doped samples and this value is far smaller than the ratio of 0.7 for the B-doped and BN-doped samples. Additionally, the full width at half maximum (FWHM) of the $G$ band for the B- and BN-doped samples $\left(32\right.$ and $\left.29 \mathrm{~cm}^{-1}\right)$ is larger than that for the undoped and N-doped samples (25 and $17 \mathrm{~cm}^{-1}$ ). Therefore, arc-discharge evaporation of graphite in helium or nitrogen atmospheres results in the formation of carbon nanoparticles with graphite-like structure. At that, the N-doped sample has the most perfect $s p^{2}$-hybridized network among all the studied samples. The addition of amorphous boron in the synthesis strongly increases disorder in graphitic shells. Furthermore, for the B-doped and BN-doped samples we observe upshifts of D band by ca. $7 \mathrm{~cm}^{-1}$ and $\mathrm{G}$ band by $c a .11 \mathrm{~cm}^{-1}$, which are more probably attributed to the hole doping [34].

Overall XPS spectra of the samples revealed the signals from carbon, oxygen, nitrogen and boron. The intensity ratios of these lines were used to evaluate the surface concertation of elements (Table 1). The sample produced in nitrogen atmosphere contains $c a$. 0.5 at\% of nitrogen. The temperature of the electric arc near the anode reaches $4000-5000{ }^{\circ} \mathrm{C}$ [35], that is enough for the nitrogen gas dissociation, which occurred at about $3500^{\circ} \mathrm{C}$ [36]. The content of boron in the B-doped sample was also $\mathrm{ca}$. $0.5 \mathrm{at} \%$. The fraction of both boron and nitrogen increases to $c a .1$ at\% in the BN-doped sample. Since the XPS signal from oxygen can appear not only from the samples but also from copper holder, the oxygen content is difficult to compare.

The XPS lines and NEXAFS spectra were used to determine the chemical states of the elements. The $C 1 s$ XPS spectra of the samples are presented by asymmetric peaks located at $284.5 \mathrm{eV}$ (Fig. 4a), corresponding to the $s p^{2}$-hybridized carbon [37]. The main difference between the spectra is the width of the peak, responsible for atomic 


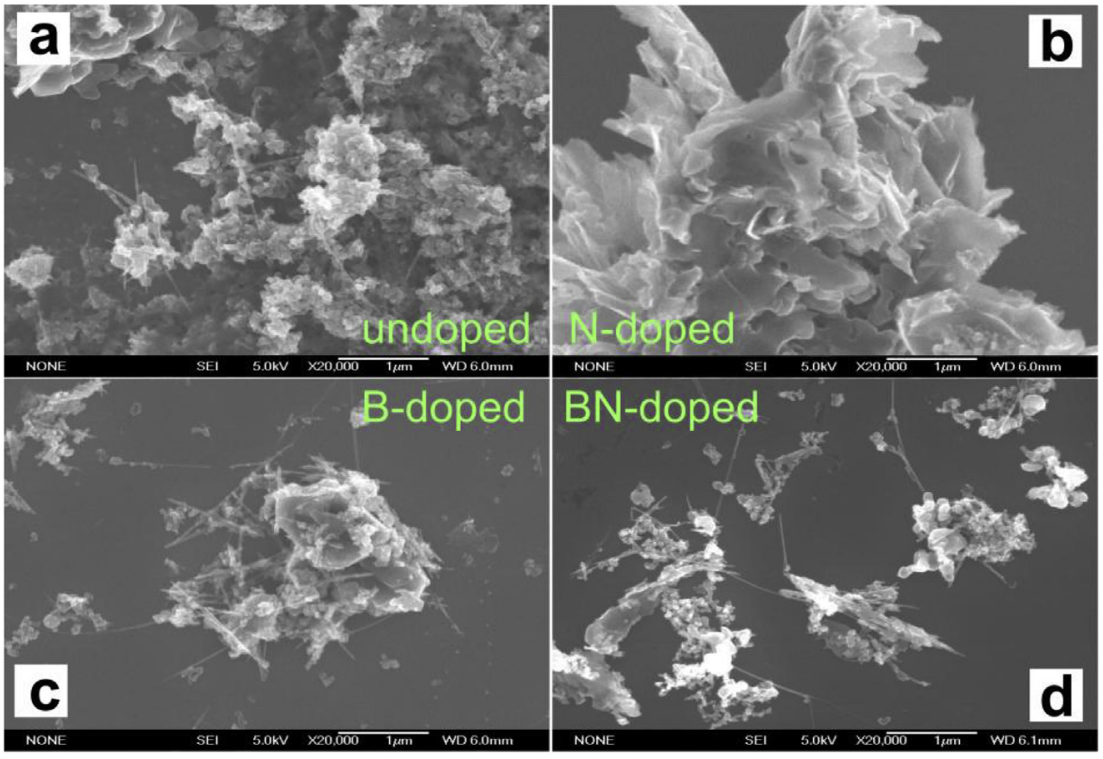

Fig. 1. SEM images of undoped (a), N-doped (b), B-doped (c), and BN-doped (d) samples. (A colour version of this figure can be viewed online.)

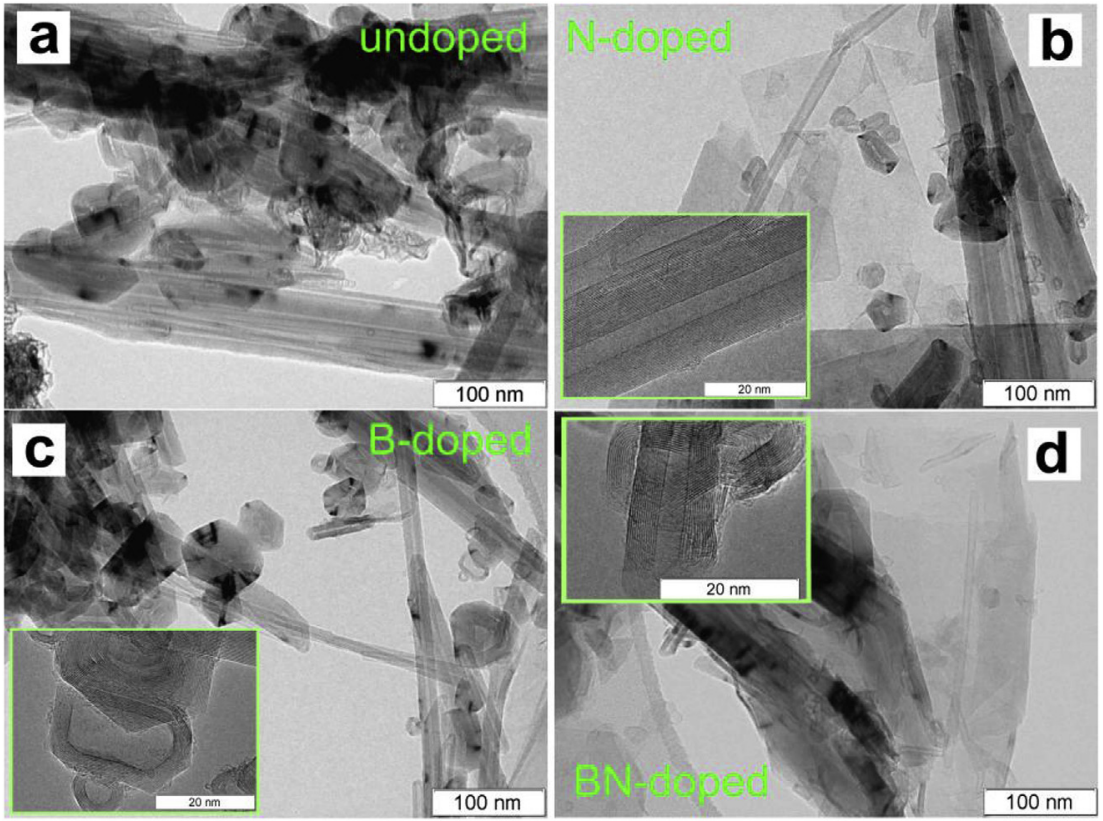

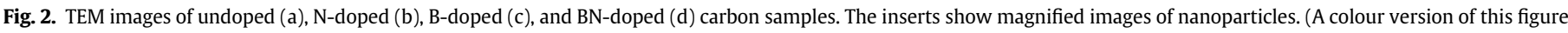
can be viewed online.)

ordering in graphitic layers. The FWHM value for the undoped, Ndoped, B-doped, and BN-doped samples is equal to $0.8,1.0,1.1$, and $1.5 \mathrm{eV}$, respectively. The increase of FWHM values with doping indicates disorder in the graphitic network. A low-energy shift of the $C$ $1 s$ spectrum of the B-doped sample by $0.3 \mathrm{eV}$ as compared to other spectra could be due to the $p$-type doping effect [38].

The C K-edge NEXAFS spectra of all samples show two main peaks at 285.4 and $293.0 \mathrm{eV}$ (Fig. $4 \mathrm{~b}$ ) assigned to $1 \mathrm{~s} \rightarrow \pi^{*}$ and $1 \mathrm{~s} \rightarrow \sigma^{*}$ transitions, respectively [37,39]. The sharpness of these resonances is a sign of good atomic ordering within the graphitic layers in carbon particles. Since the probing depth of NEXAFS is $c a .10 \mathrm{~nm}$, the higher intensity of the $\pi^{*}$ resonance for the $\mathrm{N}$-doped sample evidences the higher crystallinity of inner layers of the nanoparticles. While surface-sensitive XPS technique indicates that nanoparticles in the undoped sample have the better quality of external layers. A weak peak at $288.6 \mathrm{eV}$ labeled $c$ is likely contributed by carbon bonded with oxygen. The spectra of the Bdoped and BN-doped samples have a low-energy peak $a$ at $284.5 \mathrm{eV}$ associated with downshift of the Fermi level due to transfer of electron density from carbon cagesto incorporated boron. This agrees well with the upshifts of the D and G Raman bands. The spectrum of the BN-doped sample exhibits a lowering of the $\pi^{*}$ resonance and increase of relative intensities of the peaks $b$ and $c$ located at 287.8 and $288.6 \mathrm{eV}$, respectively (Fig. 4b). These peaks could be attributed to the formation of covalent bonds between carbon and boron, as well as carbon and nitrogen (B-C-N species). 


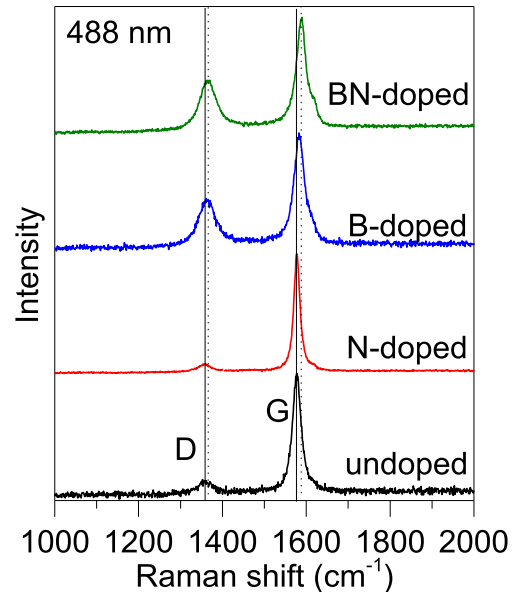

Fig. 3. Raman spectra of undoped, B-doped, N-doped, and BN-doped carbon samples. (A colour version of this figure can be viewed online.)

Table 1

Concentration of carbon, nitrogen, boron, and oxygen in the samples estimated by XPS.

\begin{tabular}{lllll}
\hline Sample & \multicolumn{4}{l}{ Element concentration, at\% } \\
\cline { 2 - 5 } & $C$ & $N$ & $B$ & $O$ \\
\hline undoped & 96 & - & - & 4 \\
N-doped & 95 & 0.5 & - & 4.5 \\
B-doped & 96 & - & 0.5 & 3.5 \\
BN-doped & 92 & 1 & 1 & 6 \\
\hline
\end{tabular}

The N $1 s$ XPS spectrum of the N-doped sample was fitted by two components positioned at 401.5 and $398.5 \mathrm{eV}$ (Fig. 5a). The former component denoted $\mathrm{N}_{\mathrm{gr}}$ corresponds to the nitrogen atoms replacing the carbon atoms in a hexagonal lattice (i.e., graphitic $\mathrm{N}$ ) $[40,41]$. The latter wide component denoted $\mathrm{N}_{\text {def }}$ could be assigned to the nitrogen atoms, being different from the graphitic one. According to the previous works, the $\mathrm{N} 1 \mathrm{~s}$ line at $398.5 \mathrm{eV}$ is usually attributed to pyridine-like nitrogen $[39,40]$. However, we do not expect that the dangling bonds could persist under the extremely high temperature of the electric arc. Most likely, that the $\mathrm{N}_{\mathrm{def}}$ peak corresponds to the two-fold coordinated nitrogen atoms located on the edges of graphitic particles or three-fold coordinated $\mathrm{N}$ atoms in topological defects.

The N K-edge NEXAFS spectrum of the N-doped sample shows two states before the $\sigma^{*}$-edge [42]. Taking into account the XPS data, a peak at $402 \mathrm{eV}$ is assigned to the $\pi^{*}$ states of graphitic $\mathrm{N}$ $\left(\mathrm{N}_{\mathrm{gr}}\right)$, while a sharp peak at $399.7 \mathrm{eV}$ is attributed to $\mathrm{N}_{\text {def. }}$. According to quantum-chemical studies, the insertion of nitrogen in carbon pentagons near the nanotube caps is energetically more preferable than in the nanotube body $[43,44]$. The pentagons are needed to close the nanotube ends and to produce spherical or polyhedral nanoparticles, which are abundant in the arc-produced samples (Fig. 2).

The $\mathrm{N} 1 \mathrm{~s}$ spectrum of the BN-doped sample also has two peaks denoted as $\mathrm{N}_{\text {gr }}$ and $\mathrm{N}_{\text {def. }}$ (Fig. 5a). However, nitrogen bonded to boron also contributes to the latter peak [45]. The occurrence of BN species in this sample is supported by an intense peak at $401.4 \mathrm{eV}$ in the $\mathrm{N} K$-edge spectrum [42]. The component arisen from BN species is located at $190.8 \mathrm{eV}$ in the B $1 s$ XPS spectrum (Fig. 6a) and $192.0 \mathrm{eV}$ in the NEXAFS B K-edge spectrum (Fig. 6b) $[45,46]$. Further, lowenergy weak peaks in the XPS B $1 s$ spectrum (ca. $186.7 \mathrm{eV}$, Fig. 6a) and NEXAFS B K-edge spectrum (ca. $189.0 \mathrm{eV}$, Fig. 6b) of the $\mathrm{BN}$-doped sample indicate that boron atoms interact also with carbon atoms. Additionally, the spectra show formation of B-O species in the BN-doped and B-dopes samples.

Segregation of $s p^{2}$-hybridized carbon and BN phases has been observed for arc-produced samples previously [13,14,16,21]. This was associated with a weaker coupling between $\mathrm{C}$ and $\mathrm{B}$ or $\mathrm{C}$ and $\mathrm{N}$ in comparison with $\mathrm{C}-\mathrm{C}$ and $\mathrm{B}-\mathrm{N}$ bonding $[16,47]$ and stabilization of C-BN heterojunctions [47]. We suppose that obtained BN-doped nanoparticles contain BN-domains embedded into $s p^{2}$-hybridized carbon network. This is different from the structure of N- and Bdoped nanoparticles, which contain point substitutional defects. The regions of carbon and BN in nanoparticles doped with BN are probably linked through C - B bonds. Actually, analysis of the XPS data shows that the fraction of these species is about two times greater than the content of $\mathrm{C}-\mathrm{N}$ species $\left(n_{\mathrm{C}-\mathrm{B}}=0.30\right.$ at\% and $n_{\mathrm{C}-}$ $\mathrm{N}=0.18$ at $\%$.

It should be noted that we did not observe the peak at $405 \mathrm{eV}$ in the XPS N 1s spectra and the split resonance at $401 \mathrm{eV}$ in the NEXAFS N K-edge spectra from molecular nitrogen [48-50]. Thus, in contrast to $\mathrm{N}$-doped MWCNTs produced by chemical vapor
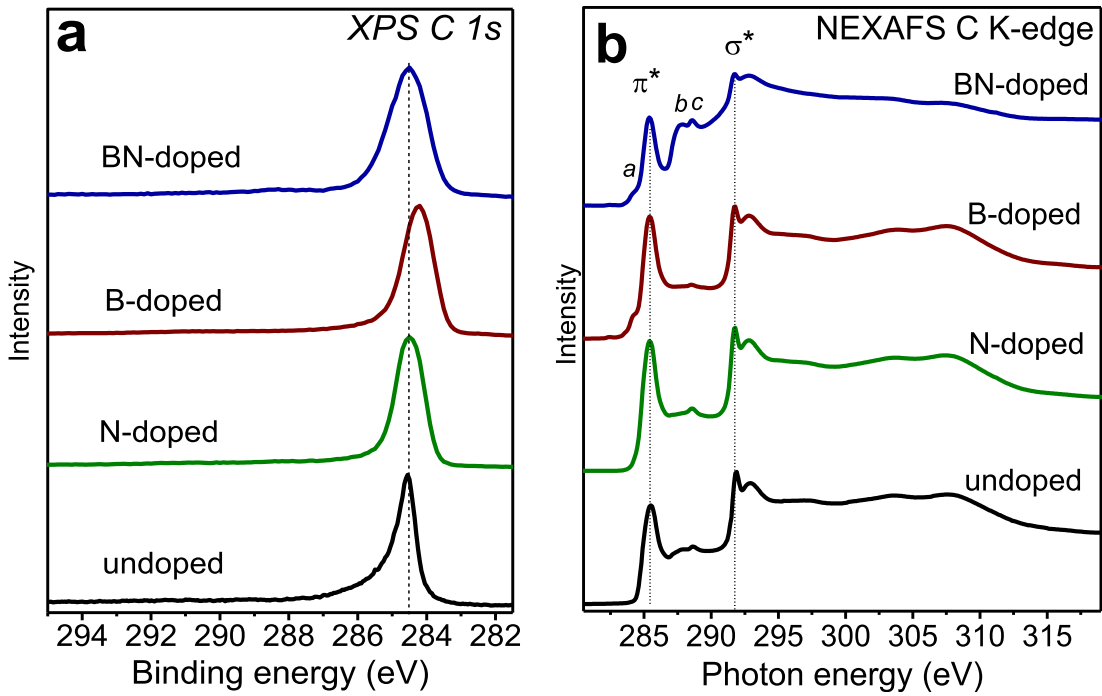

Fig. 4. XPS C $1 s$ spectra (a) and NEXAFS C K-edge spectra (b) of undoped, N-doped, B-doped, and BN-doped samples. (A colour version of this figure can be viewed online.) 

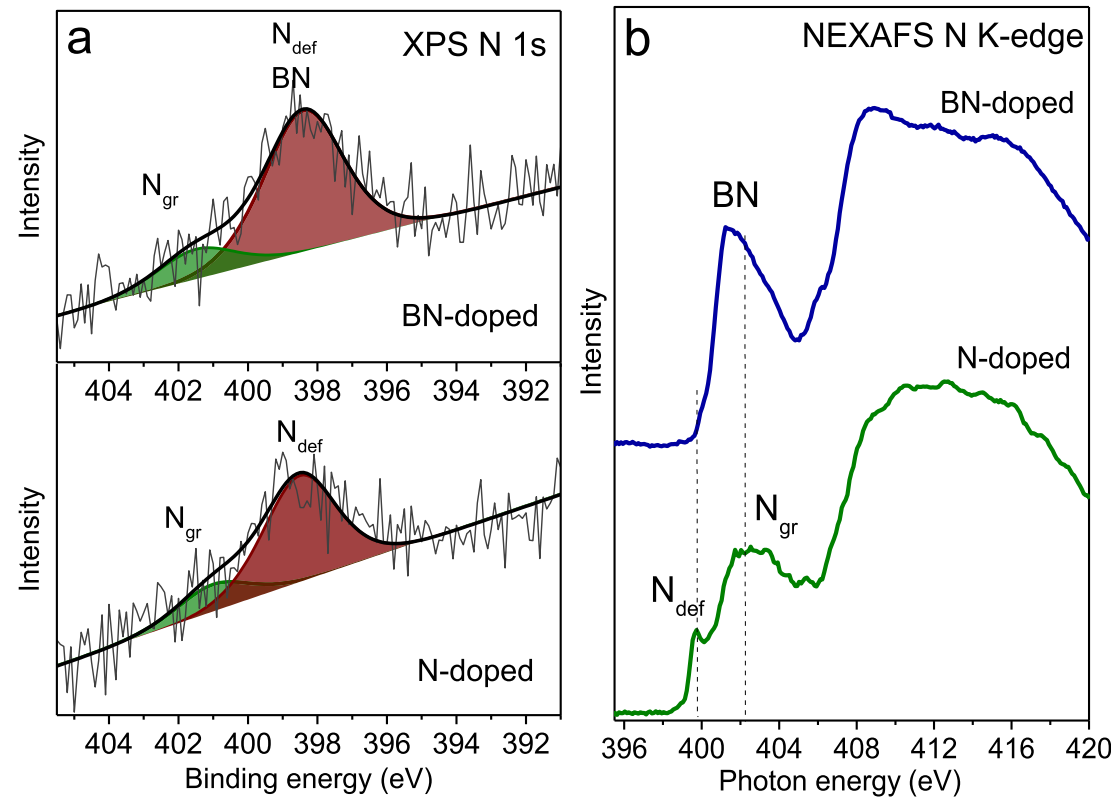

Fig. 5. N 1s XPS (a) and N K-edge NEXAFS spectra (b) of N-doped and BN-doped samples. (A colour version of this figure can be viewed online.)

deposition technique using $\mathrm{N}$-containing precursors, the arcproduced $\mathrm{N}$-doped and $\mathrm{BN}$-doped carbon samples do not contain encapsulated $\mathrm{N}_{2}$ molecules.

\subsection{Transport properties}

Fig. 7a shows the Hall voltage $V_{H} v s$ magnetic field $H$ for the $\mathrm{N}$ doped sample (left $V_{H}$ scale) and the B-doped sample (right $V_{H}$ scale). The slope of the $V_{H^{-}} H$ dependence is positive for the B-doped sample and negative for the $\mathrm{N}$-doped sample indicating that holes and electrons, respectively, are predominant charge carriers. The $p$ type conductivity of the B-doped sample agrees with the XPS and NEXAFS data. The Hall measurements allow us to determine the concentration of charge carriers. Assuming that only one type of carriers is present in the $\mathrm{N}$-doped sample, their concentration is about $310^{18} \mathrm{~cm}^{-3}$. The donor states are usually assigned to graphitic nitrogen. We suggest that these atoms are likely embedded into graphitic plates, which are abundant in the $\mathrm{N}$-doped sample. The hole concentration of the B-doped sample is about $10^{21} \mathrm{~cm}^{-3}$. Thus, even small content of boron atoms bonded with carbon is enough for the substantial donation of free holes to the electronic system of carbon nanoparticles. It is worth noting that the carrier concentration in the B-doped sample is about two orders of magnitude higher than in the case of N-doping.

Fig. $7 \mathrm{~b}$ shows the temperature dependences of the conductivity normalized to room temperature value $\sigma(T) / \sigma\left(T_{r}\right)$ for all samples. In the case of the undoped sample, the conductivity increases from 85 to $163 \mathrm{~S} / \mathrm{cm}$ as the temperature rises from 4.2 to $293 \mathrm{~K}$. The doped
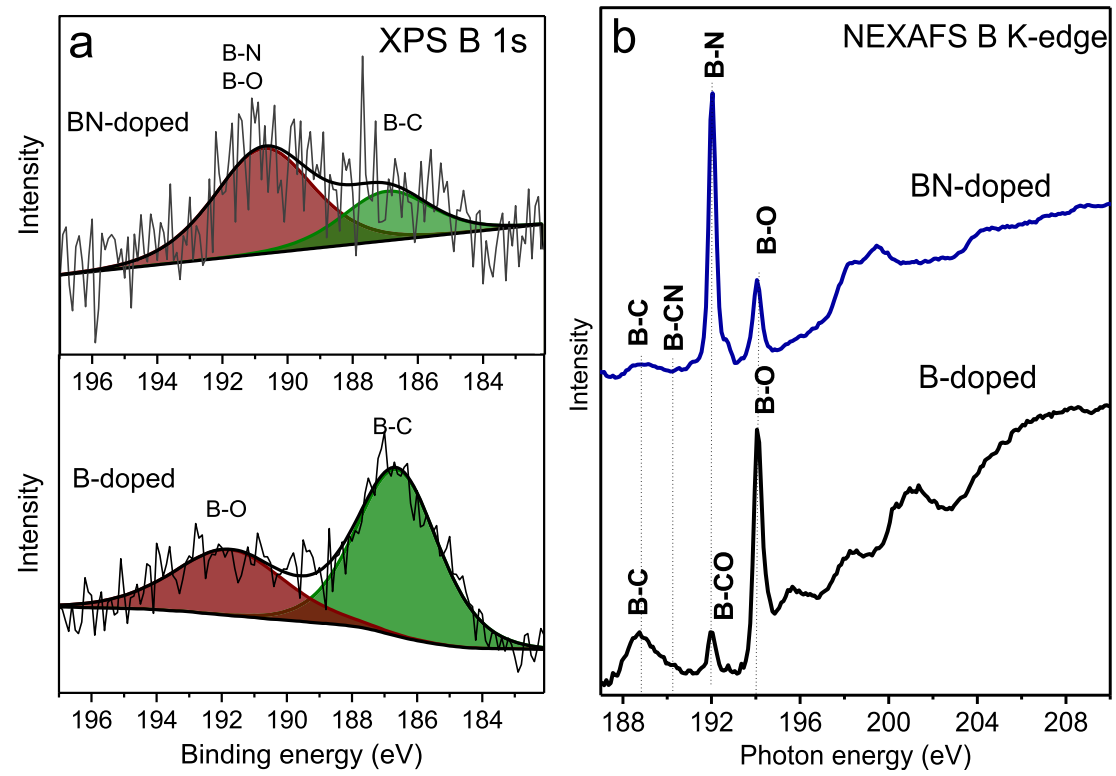

Fig. 6. B $1 s$ XPS (a) and NEXAFS B K-edge spectra (b) of B-doped and BN-doped samples. (A colour version of this figure can be viewed online.) 
samples have the higher conductivity. $\sigma\left(T_{r}\right)$ values are 184 and $314 \mathrm{~S} / \mathrm{cm}$ for the $\mathrm{N}$ - and B-doped samples, respectively. These values are typical for arc-discharge products [24,25]. The conductivity of the BN-doped sample is significantly higher $(493 \mathrm{~S} / \mathrm{cm})$ than that for the samples doped with nitrogen or boron solely. This enhancement can be assigned to the formation of B-terminated BNdomains in carbon network. The extended B-rich interfaces donate holes in the electronic system of carbon nanoparticle more effectively than isolated boron atoms. This indicates the synergetic effects of simultaneous nitrogen and boron incorporation into the carbon network of obtained nanoparticles.

The conductivity curves of all samples have two regions with different behavior. At temperatures above $100 \mathrm{~K}$, the conductivity can be expressed by any exponential behavior. In particular, approximation of the conductivity by the $\exp (-E / T)$ reveals that doping decreases the tunneling barrier $E$. For the undoped and $\mathrm{N}$ doped samples, $E$ is quite high (about 153 and $93 \mathrm{~K}$, respectively), while it drops to about 4.5 and $7 \mathrm{~K}$ for the B- and BN-doped samples. Besides, both two- and three-dimensional variable-range hopping transports (as well as their combinations) are suitable to approximate the conductivity dependencies. It is likely, that highly nonhomogeneous structure of the samples causes a simultaneous movement of electrons (or holes) along different paths. The $\sigma(T)$ dependences of the undoped and $\mathrm{N}$-doped samples can also be fitted by a power law $T^{1.5}$. Such temperature behavior is typical for doped semiconductors, whose conductivity is determined by impurity scattering [51]. Therefore, both the tunneling between neighboring nanoparticles and the intrinsic conductivity of nanotubes and graphitic plates contribute to the transport properties of the samples containing well-ordered graphitic layers (undoped and $\mathrm{N}$-doped). The addition of boron into electric arc enhances disorder in carbon network that, in particular, is exhibited as the formation of many small polyhedral nanoparticles. On the other hand, strong hole doping of the samples enhances a coupling between neighboring nanoparticles. As a result, conductivities of the B-doped and $\mathrm{BN}$-doped samples weakly depend on the temperature and have a metallic behavior above $100 \mathrm{~K}$.

Below $100 \mathrm{~K}$, the conductivity of all samples follows a logarithmic law ( $\ln T$, Fig. 7c) due to the contributions of quantum effects inherent to the disordered systems [52]. For such systems, the transport mechanism can be described by a diffuse conductance within the concept of weak localization (WL). When two paths of an electron wave function form a closed loop, they become phase coherent and the constructive interference tends to localize electrons.

The contribution of $\mathrm{WL}$ to low-temperature transport is confirmed by the MC measurements, which are sensitive to the crystallinity of nanoparticles, their shapes and sizes (Fig. 8). The undoped, B-doped and BN-doped samples have a positive dependence of MC (Fig. 8a). When electrons are localized, magnetic field breaks the constructive quantum interference. The higher the magnetic field, the weaker the localization effects, the higher the conductivity. MC follows quadratic law $\mathrm{H}^{2}$ at low fields and increases as $\ln H$ at higher field (Fig. $8 \mathrm{~b}-\mathrm{d}$ ). These asymptotes are specific for materials, whose charge transport is governed by twodimensional WL effects, which is widely used to explain positive MC in disordered thin metal films [53], amorphous carbon materials [54,55], disordered graphite [56], carbon nanotubes [57], graphene $[58,59]$. Although the electron-electron interaction effect causes the same dependence of $\mathrm{MC}(H)$ [52], we should rule it out due to rather small values of the magnetic field. On the other hand, the arc-discharge products are nonhomogeneous materials, and electrons are diffusively scattered at boundaries of crystallites that restricts their free paths [60]. The diffusion scattering and WL effects will make comparable contributions to the macroscopic conductivity of nanostructure with a linear dimension of about or less than $10 \mathrm{~nm}$ [61]. The magnetic field induces extension of the mean free path, thus increasing the conductivity of the nanoparticles as $H^{2}$ [62].

The field dependence of MC has qualitatively different behavior for the N-doped sample (Fig. 8a). The conductivity increases from zero to $0.3 \mathrm{~T}$ and then a negative contribution to $\mathrm{MC}$ is observed $\left(\sim-H^{2}\right.$, Fig. 8 e). Such behavior could be related to the ordinary Lorentz cyclic motion of electrons in magnetic field [63]. Similar crossover from positive to negative MC was found for bulk graphite materials with different particle sizes [61]. In particular, the conductivity of graphite composed of 30-nm particles increases with the magnetic field due to WL effects and diffuse scattering at the boundaries of crystallites. When the size of the particles reaches several micrometers, the conductivity decreases as $\sim-H^{2}$. Therefore, we suppose that the crossover in the $\sigma(H)$ dependence of the $\mathrm{N}$ doped sample is due to the compensation of quantum transport
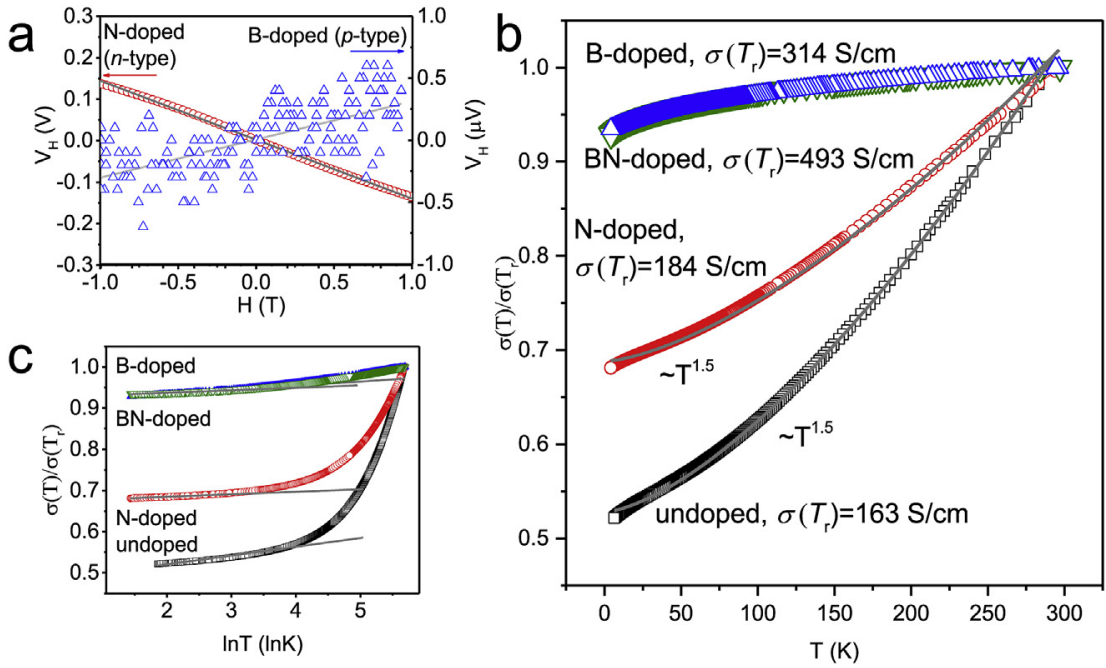

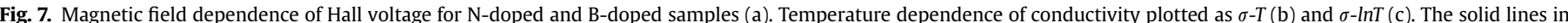

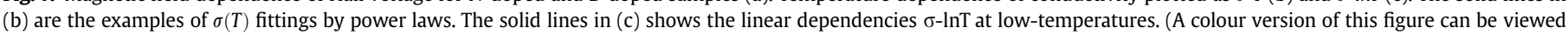
online.) 

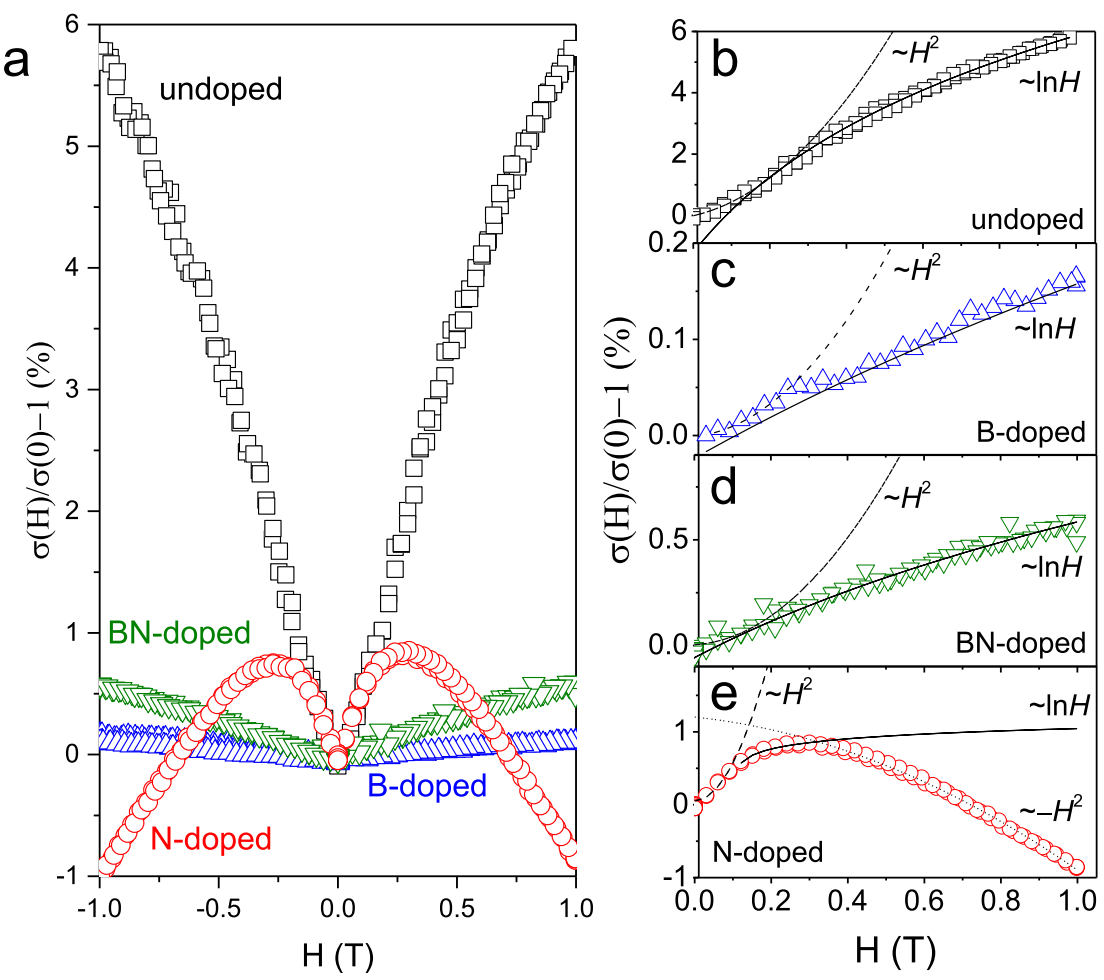

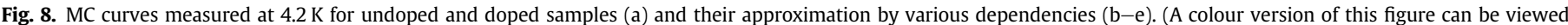
online.)

through small nanoparticles by ordinary cyclic motion of electrons through graphitic plates. Other samples (undoped, B-doped, and BN-doped) mainly consist of nanotubes and nanoparticles with a small fraction of graphitic plates supporting the ordinary MC effect. As the result, these samples showed the charge transport properties characteristic of the disordered systems.

\section{Conclusion}

Multicomponent carbon samples doped with nitrogen and/or boron with a concertation of foreign elements less than 1 at\% have been synthesized by the arc-discharge method using amorphous boron and nitrogen gas as dopant sources. SEM and TEM methods have found MWCNTs, polyhedral nanoparticles and graphitic plates in the samples. Among the synthesized samples, the $\mathrm{N}$-doped material is distinguished by the fact that it comprised a large fraction of the plates. As shown by Raman scattering, XPS and NEXAFS, this material also had a high graphitization degree comparable to the other samples. The B- and BN-doped samples were enriched by small nanoparticles and short MWCNTs. It was shown that arc-discharge evaporation of a boron-stuffed graphite electrode enhanced disorder in a honeycomb carbon lattice. A comprehensive characterization revealed the presence of $\mathrm{C}-\mathrm{N}$ and $\mathrm{C}-\mathrm{B}$ bonds confirming the incorporation of dopants in the lattice. That induced either electron or hole doping depending on whether nitrogen or boron has participated in arc-discharge process. We suggest that the co-doped sample also showed the hole doping due to the preferable involvement of nitrogen in the formation of B-N bonds, while residual boron atoms and boron atoms located at the C-BN heterojunctions acted as electron acceptors. Our data suggested that the charge carrier concentration is a dominant factor in the manifestation of semiconducting or metallic conductivity behavior of arc-discharge carbon samples. From the MC measurements, we found that both quantum correction and diffusive scattering at the crystallite boundaries govern the lowtemperature charge transport properties of the undoped, Bdoped, and BN-doped samples. Above some critical value of magnetic field, the opposite trend in the conductivity was found for the $\mathrm{N}$-doped samples. It is supposed that the dominant mechanism responsible for compensation of the conductivity increase is a cyclotron motion of charges on surfaces of graphitic plates. Probably, either size of graphitic plates or their crystallinity are not enough to countervail the positive MC effects in other samples. The found relationships between synthesis conditions, morphology and conductivity of arc produced materials opens a new route toward controllable and easy to use technology of either $p$ - or $n$-type doped carbon components fabrication. Their transport properties and carrier concentrations could vary within the same process, just adding either the amorphous boron or nitrogen gas, or both, to the arc discharge.

\section{Acknowledgment}

This work was done with the support of RFBR (grant 17-5204077), BRFFR (grant F17RM-068) and H2020 RISE 734164 Graphene 3D. The work was partially supported by the bilateral Program "Russian-German Laboratory at BESSY II" in the part of XPS and NEXAFS measurements. The authors thank Mrs. L. I. Nasonova for the syntheses, Mr. A. V. Ishchenko for the TEM measurements and Dr. I. P. Asanov for the XPS measurement of the undoped sample. We are grateful to Prof. V. A. Osipov and Dr. V. L. Katkov for fruitful discussion. O.V.S., L.G.B., D.S.B., P.P.K., and A.V.O. are thankful for support by Tomsk State University Competitiveness Improvement Program. O.V.S. thanks Scholarship of the President of the Russian Federation (SP-3530.2016.1). 


\section{References}

[1] S. Iijima, Helical microtubules of graphitic carbon, Nature 354 (1995) 56-58.

[2] X. Zhao, M. Ohkohchi, S. Inoue, T. Suzuki, T. Kadoya, Y. Ando, Large-scale purification of single-wall carbon nanotubes prepared by electric arc discharge, Diam. Relat. Mater. 15 (4-8) (2006), 1098-02.

[3] N.I. Alekseyev, G.A. Dyuzhev, Fullerene formation in an arc discharge, Carbon 41 (7) (2003) 1343-1348.

[4] F. Liang, V. Shimizu, M. Tanaka, S. Choi, T. Watanabe, Selective preparation of polyhedral graphite particles and multi-wall carbon nanotubes by a transferred arc under atmospheric pressure, Diam. Relat. Mater. 30 (2012) 70-76.

[5] K.S. Subrahmanyam, L.S. Panchakarla, A. Govindaraj, C.N.R. Rao, Simple method of preparing graphene flakes by an arc-discharge method, J. Phys. Chem. C 113 (11) (2009) 4257-4259.

[6] T. Nakajima, M. Koh, T. Katsube, Structure, chemical bonding and electrochemical behavior of heteroatom-substituted carbons prepared by arc discharge and chemical vapor deposition, Solid State Sci. 2 (1) (1998) 17-29.

[7] A.V. Okotrub, L.G. Bulusheva, A.I. Romanenko, A.L Chuvilin, N.A. Rudina, Y.V. Shubin, et al., Anisotropic properties of carbonaceous material produced in arc discharge, Appl. Phys. A 72 (4) (2001) 481-486.

[8] O. Stephan, P.M. Ajayan, C. Colliex, Ph Redlich, J.M. Lambert, P. Bernier, et al., Doping graphitic and carbon nanotube structures with boron and nitrogen, Science 266 (5191) (1994) 1683-1685.

[9] M. Scardamaglia, C. Struzzi, F.J.A. Rebollo, P.D. Marco, P.R. Mudimela, J.F. Colomer, et al., Tuning electronic properties of carbon nanotubes by nitrogen grafting: Chemistry and chemical stability, Carbon 83 (2015) 118-127.

[10] B. Ruelle, A. Felten, J. Ghijsen, W. Drube, R.L. Johnson, D. Liang, R. Erni, G. Van Tendeloo, S. Peeterbroeck, P. Dubois, T. Godfroid, M. Hecq, C. Bittencourt, Functionalization of MWCNTs with atomic nitrogen, Micron 40 (1) (2009) 85-88.

[11] A.G. Kurenya, L.G. Bulusheva, I.P. Asanov, O.V. Sedelnikova, A.V. Okotrub, Field emission properties of aligned $\mathrm{CN}_{\mathrm{x}}$ nanotube arrays synthesized by pyrolysis of a ferrocene/acetonitrile aerosol at different temperatures, Phys. Status Solidi B 252 (11) (2015) 2524-2529.

[12] Yu.V. Fedoseeva, A.V. Okotrub, I.P. Asanov, D.V. Pinakov, G.N. Chekhova, V.A. Tur, P.E. Plyusnin, D.V. Vyalikh, L.G. Bulusheva, Nitrogen inserting in fluorinated graphene via annealing of acetonitrile intercalated graphite fluoride, Phys. Status Solidi B 251 (12) (2014) 2530-2535.

[13] Ph. Redlich, J. Loeffler, P.M. Ajayan, J. Bill, F. Aldinger, M. Rühle, B-C-N nanotubes and boron doping of carbon nanotubes, Chem. Phys. Lett. 260 (1996) 465-470.

[14] K. Suenaga, C. Colliex, N. Demoncy, A. Loiseau, H. Pascard, F. Willaime, Synthesis of nanoparticles and nanotubes with well-separated layers of boron nitride and carbon, Science 278 (5338) (1997) 653-655.

[15] M. Terrones, W.K. Hsu, S. Ramos, R. Castillo, H. Terrones, The role of boron nitride in graphite plasma arcs, Fullerene Sci. Technol. 6 (5) (1998) 787-800.

[16] K. Suenaga, F. Willaime, A. Loiseau, C. Colliex, Organisation of carbon and boron nitride layers in mixed nanoparticles and nanotubes synthesized by arc discharge, Appl. Phys. A 68 (3) (1999) 301-308.

[17] W.K. Hsu, S. Firth, Ph Redlich, M. Terrones, H. Terrones, Y.Q. Zhu, et al., Borondoping effects in carbon nanotubes, J. Mater. Chem. 10 (6) (2000) 1425-1429.

[18] R. Droppa, P. Hammer, A.C.M. Carvalho, M.C. dos Santos, F. Alvarez, Incorporation of nitrogen in carbon nanotubes, J. Non-Cryst. Solids 299-302 (2) (2002) 874-879.

[19] M. Glerup, J. Steinmetz, O. Stéphan, S. Enouz, A. Loiseau, S. Roth, et al., Synthesis of N-doped SWNT using the arc-discharge procedure, Chem. Phys. Lett. $387(1-3)$ (2004) 193-197.

[20] F. Piazza, J.E. Nocua, A. Hidalgo, J. de Jesús, R. Velázquez, B.L. Weiss, et al., Formation of boron carbonitride nanotubes from in situ grown carbon nanotubes, Diam. Rel. Matter. 14 (2005) 965-969.

[21] S.M.C. Vieira, O. Stéphan, D.L. Caroll, Effect of growth conditions on B-doped carbon nanotubes, J. Mater. Res. 21 (12) (2006) 3058-3064.

[22] B. Wang, Y. Ma, Y. Wu, N. Li, Y. Huang, Y. Chen, Direct and large scale electric arc discharge synthesis of boron and nitrogen doped single-walled carbon nanotubes and their electronic properties, Carbon 47 (8) (2009) 2112-2142.

[23] A.B. Belgacem, I. Hinkov, S.B. Yahia, O. Brinza, S. Farhat, Arc discharge boron nitrogen doping of carbon nanotubes, Mat. Today Com. 8 (2016) 183-195.

[24] A.O. Plyushch, A.A. Sokol, K.N. Lapko, P.P. Kuzhir, Yu.V. Fedoseeva, A.I. Romanenko, O.B. Anikeeva, L.G. Bulusheva, A.V. Okotrub, Electromagnetic properties of phosphate composite materials with boron-containing carbon nanotubes, Phys. Solid State 56 (12) (2014) 2537-2542.

[25] M.V. Shuba, D.I. Yuko, P.P. Kuzhir, S.A. Maksimenko, G.G. Chigir, A.N. Pyatlitski, et al., Localized plasmon resonance in boron-doped multiwalled carbon nanotubes, Phys. Rev. B 97 (2018), 205427.

[26] C.P. Ewels, M. Glepur, Nitrogen doping in carbon nanotubes, J. Nanosci. Nanotechnol. 5 (9) (2005) 1345-1363.

[27] L.S. Panchakarla, A. Govindaraj, C.N.R. Rao, Boron- and nitrogen-doped carbon nanotubes and graphene, Inorg. Chim. Acta. 363 (15) (2010) 4163-4174.

[28] D. Jana, C.-L. Sun, L.-C. Chen, K.-H. Chen, Effect of chemical doping of boron and nitrogen on the electronic, optical, and electrochemical properties of carbon nanotubes, Chen. Prog. Mater. Sci. 58 (5) (2013) 565-635.

[29] A.M. Nemilentsau, M.V. Shuba, G.Ya. Slepyan, P.P. Kuzhir, S.A. Makximenko, Substitutional doping of carbon nanotubes to control their electromagnetic characteristics, Phys. Rev. B 82 (2010), 235424.
[30] M.A. Kanygin, O.V. Sedelnikova, I.P. Asanov, L.G. Bulusheva, A.V. Okotrub,, P.P. Kuzhir, et al., Effect of nitrogen doping on the electromagnetic properties of carbon nanotube-based composites, J. Appl. Phys. 113 (14) (2013) 144315.

[31] A.V. Okotrub, Y.V. Shevtsov, L.I. Nasonova, D.E. Sinyakov, A.V. Chuvilin, A.K. Gutakovskii, et al., Arc-discharge synthesis of single-shell carbon particles, Inorg. Mater. 32 (8) (1996) 858-861.

[32] P.G. Collins, K. Bradley, M. Ishigami, A. Zettl, Extreme oxygen sensitivity of electronic properties of carbon nanotubes, Science 287 (5459) (2000) 1801-1804.

[33] A.C. Ferrari, Raman spectroscopy of graphene and graphite: disorder, electron-phonon coupling, doping and nonadiabatic effects, Solid State Commun. $143(1-2)(2007)$ 47-57.

[34] A. Jorio, M. Dresselhaus, R. Saito, G.F. Dresselhaus, Raman Spectroscopy in Graphene Related Systems, Wiley-VCH, Weinheim, Germany, 2011.

[35] G. Raniszewski, Temperature measurements in arc-discharge synthesis of nanomaterials dedicated for medical applications, Eur. Phys. J. Appl. Phys. 61 (2) (2013) 24311.

[36] J.M. Hendrie, Dissociation energy of $\mathrm{N}_{2}$, J. Chem. Phys. 22 (9) (1954) 1503-1507.

[37] N. Tran, M. Wilson, A. Milev, J. Bartlett, R. Lamb, D. Martin, et al., Photoemission and absorption spectroscopy of carbon nanotube interfacial interaction, Adv. Colloid Interface Sci. 145 (1-2) (2009) 23-41.

[38] L.G. Bulusheva, A.V. Okotrub, E. Flahaut, I.P. Asanov, P.N. Gevko, V.O. Koroteev, et al., Bromination of double-walled carbon nanotubes, Chem. Mater. 24 (14) (2012) 2708-2715.

[39] Yu.V. Fedoseeva, G.A. Pozdnyakov, A.V. Okotrub, M.A. Kanygin, Yu.V. Nastaushev, O.Y. Vilkov, et al., Effect of substrate temperature on the structure of amorphousoxygenated hydrocarbon films grown with a pulsed supersonicmethane plasma flow, Appl. Surf. Sci. 385 (1) (2016) 464-471.

[40] M. Scardamaglia, M. Amati, B. Llorente, P. Mudimela, J.-F. Colomer, J. Ghijsen, et al., Nitrogen ion casting on vertically aligned carbon nanotubes: tip and sidewall chemical modification, Carbon 77 (2014) 319-328.

[41] T. Susi, T. Pichler, P. Ayala, X-ray photoelectron spectroscopy of graphitic carbon nanomaterials doped with heteroatoms, Beilstein J. Nanotechnol. 6 (2015) 177-192.

[42] Yu.V. Fedoseeva, M.L. Kosinova, S.A. Prokhorova, I.S. Merenkov, L.G. Bulusheva, A.V. Okotrub, et al., X-ray spectroscopic study of the electronic structure of boron carbonitride films obtained by chemical vapor deposition on $\mathrm{Co} / \mathrm{Si}$ and $\mathrm{CoO}_{\mathrm{x}} / \mathrm{Si}$ substrates, J. Struct. Chem. 53 (4) (2012) 690-698.

[43] L.G. Bulusheva, O.V. Sedelnikova, A.V. Okotrub, Substitutional sites of nitrogen atoms in carbon nanotubes and their influence on field-emission characteristics, Int. J. Quant. Chem. 111 (11) (2011) 2696-2704.

[44] O.V. Sedelnikova, L.G. Bulusheva, A.V. Okotrub, Graphitic and pyridinic nitrogen in carbon nanotubes: energetic and polarization aspects, J. Nanophotonics 10 (1) (2015) 012510.

[45] M. Petravic, R. Peter, L.-J. Fan, Y.-W. Yang, Y. Chen, Direct observation of defects in hexagonal boron nitride by near-edge X-ray absorption fine structure and X-ray photoemission spectroscopy, Nucl. Instrum. Methods Phys. Res. A $619(1-3)(2010) 94-97$.

[46] R. Peter, A. Bozanic, M. Petravic, Y. Chen, L.-J. Fan, Y.-W. Yang, Formation of defects in boron nitride by low energy ion bombardment, J. Appl. Phys. 106 (8) (2009) 083523.

[47] X. Blasé, J.-C. Charlier, A. De Vita, R. Car, Theory of composite $\mathrm{B}_{\mathrm{X}} \mathrm{C}_{\mathrm{y}} \mathrm{N}_{\mathrm{z}}$ nanotube heterojunctions, Appl. Phys. Lett. 70 (2) (1997) 197-199.

[48] L.G. Bulusheva, A.V. Okotrub, Yu.V. Fedoseeva, A.G. Kurenya, I.P. Asanov, O.Y. Vilkov, A.A. Koor's, N. Grobert, Controlling pyridinic, pyrrolic, graphitic, and molecular nitrogen in multi-wall carbon nanotubes using precursors with different N/C ratios in aerosol assisted chemical vapor deposition, Phys. Chem. Chem. Phys. 17 (37) (2015) 23741-23747.

[49] A.V. Okotrub, M.A. Kanygin, L.G. Bulusheva, D.V. Vyalikh, X-ray absorption spectra of $\mathrm{N}_{2}$ molecules embedded into CNx nanotubes as a marker of orientation ordering of array, Fullerenes, Nanotub. Carbon Nanostruct. 18 (4-6) (2010) 551-557.

[50] L.G. Bulusheva, A.V. Okotrub, A.G. Kudashov, E.M. Pazhetnov, A.I. Boronin, D.V. Vyalikh, Encapsulation of molecular nitrogen in multiwall $\mathrm{CN}_{\mathrm{X}}$ nanotubes, Phys. Status Solidi B 244 (11) (2007) 4078-4081.

[51] H. Brooks, Theory of the electrical properties of germanium and silicon, Adv. Electron. Electron. Phys. 7 (1955) 85-182.

[52] P.A. Lee, T.V. Ramakrishnan, Disordered electronic systems, Rev. Mod. Phys. 57 (2) (1985) 287-337.

[53] G. Bergmann, Weak localization in thin films - a time-of-fight experiment with conduction electrons, Phys. Rep. 107 (1) (1984) 1-58.

[54] J.M. Wang, X.Z. Zhang, C.H. Wan, J. Vanackkn, V.V. Moshchalkov, Magnetotransport properties of undoped amorphous carbon films, Carbon 59 (2013) $278-282$.

[55] U. Rizwan, X.Z. Zhang, J.M. Wang, C.Y. Xiong, Angular magnetoresistance in semiconducting undoped amorphous carbon thin films, J. Appl. Phys. 117 (17) (2015) 174503.

[56] V. Bayot, L. Piraux, J.-P. Michenaud, J.-P. Issi, M. Lelaurain, A. Moore, Twodimensional weak localization in partially graphitic carbons, Phys. Rev. B 41 (1990) 11770-11779.

[57] J.S. Bulmer, A. Lekawa-Raus, D.G. Rickel, F.F. Balakirev, K.K. Koziol, Extreme magneto-transport of bulk carbon nanotubes in sorted electronic concentrations and aligned high performance fiber, Sci. Rep. 7 (2017) 12193.

[58] H.-C. Wu, A.N. Chaika, M.-C. Hsu, T.-W. Huang, M. Abid, M. Abid, et al., Large 
positive in-plane magnetoresistance induced by localized states at nanodomain boundaries in graphene, Nat. Commun. 8 (2017) 14453.

[59] M. Rein, N. Richter, K. Parvez, X. Feng, H. Sachdev, M. Kläui, et al., Magnetoresistance and charge transport in graphene governed by nitrogen dopants, ACS Nano 9 (2) (2015) 1360-1366.

[60] B.L. Altshuler, A.G. Aronov, Fermi-liquid theory of the electron-electron interaction effects in disordered metals, Solid State Commun. 46 (6) (1983)
$429-435$

61] X. Zhang, Q.Z. Xue, D.D. Zhu, Positive and negative linear magnetoresistance of graphite, Phys. Lett. 320 (5-6) (2004) 471-477.

[62] S. Fujita, Negative magnetoresistance in carbons and diffuse scattering at crystallite boundaries, Carbon 6 (5) (1968) 746-748.

[63] M. Kohler, Zur Magnetischen Widerstandsänderung Reiner Metalle, Ann. Phys. 424 (1-2) (1938) 211-218. 